\title{
Relation of cue to consequence in avoidance learning'
}

JOHN GARCIA AND ROBERT A. KOELLING

HARVARD MEDICAL SCHOOL AND MASSACHUSETTS GENERAL HOSPITAL

An audiovisual stimulus was made contingent upon the rat's licking at the water spout, thus making it analogous with a gustatory stimulus. When the audiovisual stimulus and the gustatory stimulus were paired with electric shock the avoidance reactions transferred to the audiovisual stimulus, but not the gustatory stimulus. Conversely, when both stimuli were paired with toxin or $x$-ray the avoidance reactions transferred to the gustatory stimulus, but not the audiovisual stimulus. Apparently stimuli are selected as cues dependent upon the nature of the subsequent reinforcer

A great deal of evidence stemming from diverse sources suggests an inadequacy in the usual formulations concerning reinforcement. Barnett (1963) has described the "bait-shy" behavior of wild rats which have survived a poisoning attempt. These animals utilizing olfactory and gustatory cues, avoid the poison bait which previously made them ill. However, there is no evidence that they avoid the "place" of the poisoning.

In a recent volume (Haley \& Snyder, 1964) several authors have discussed studies in which ionizing radiations were employed as a noxious stimulus to produce avoidance reactions in animals. Ionizing radiation like many poisons produces gastrointestinal disturbances and nausea. Strong aversions are readily established in animals when distinctively flavored fluids are conditionally paired with $x$-rays. Subsequently, the gustatory stimulus will depress fluid intake without radiation. In contrast, a distinctive environmental complex of auditory, visual, and tactual stimuli does not inhibit drinking even when the compound stimulus is associated with the identical radiation schedule. This differential effect has also been observed following ingestion of a toxin and the injection of a drug (Garcia \& Koelling, 1965).

Apparently this differential effectiveness of cues is due either to the nature of the reinforcer, i.e., radiation or toxic effects, or to the peculiar relation which a gustatory stimulus has to the drinking response, i.e., gustatory stimulation occurs if and only if the animal licks the fluid. The environmental cues associated with a distinctive place are not as dependent upon a single response of the organism. Therefore, we made an auditory and visual stimulus dependent upon the animal's licking the water spout. Thus, in four experiments reported here "bright-noisy" water, as well as "tasty" water was conditionally paired with radiation, a toxin, immediate shock, and delayed shock, respectively, as reinforcers. Later the capacity of these responsecontrolled stimuli to inhibit drinking in the absence of reinforcement was tested.

\section{Method}

The apparatus was a light and sound shielded box $(7$ in. $x 7$ in. $x$ 7 in.) with a drinking spout connected to an electronic drinkometer which counted each touch of the rat's tongue to the spout. "Brightnoisy" water was provided by connecting an incandescent lamp (5 watts) and a clicking relay into this circuit. "Tasty" water was provided by adding flavors to the drinking supply.

Each experimental group consisted of 10 rats ( 90 day old SpragueDawley males) maintained in individual cages without water, but with Purina Laboratory chow ad libidum.

The procedure was: A. One week of habituation to drinking in the apparatus without stimulation. B. Pretests to measure intake of bright-noisy water and tasty water prior to training. C. Acquisition training with: (1) reinforced trials where these stimuli were paired with reinforcement during drinking, (2) nonreinforced trials where rats drank water without stimuli or reinforcement. Training terminated when there was a reliable difference between water intake scores on reinforced and nonreinforced trials. D. Post-tests to measure intake of bright-noisy water and tasty water after training.

In the $x$-ray study an audiovisual group and a gustatory group were exposed to an identical radiation schedule. In the other studies reinforcement was contingent upon the rat's response. To insure that both the audiovisual and the gustatory stimuli received equivalent reinforcement, they were combined and simultaneously paired with the reinforcer during acquisition training. Therefore, one group serving as its own control and divided into equal subgroups, was tested in balanced order with an audiovisual and a gustatory test before and after training with these stimuli combined.

One 20-min. reinforced trial was administered every three days in the $x$-ray and lithium chloride studies. This prolonged intertrial interval was designed to allow sufficient time for the rats to recover from acute effects of treatment. On each interpolated day the animals received a 20-min. nonreinforced trial. They were post-tested two days after their last reinforced trial. The $x$-ray groups received a total of three reinforced trials, each with $54 \mathrm{r}$ of filtered $250 \mathrm{kv} x$-rays delivered in $20 \mathrm{~min}$. Sweet water ( $1 \mathrm{gm}$ saccharin per liter) was the gustatory stimulus. The lithium chloride group had a total of five reinforced trials with toxic salty water (.12 $\mathrm{M}$ lithium chloride). Nontoxic salty water (.12 M sodium chloride) which rats cannot readily distinguish from the toxic solution was used in the gustatory tests (Nachman, 1963).

The immediate shock study was conducted on a more orthodox avoidance schedule. Tests and trials were $2 \mathrm{~min}$. long. Each day for four consecutive acquisition days, animals were given two nonreinforced and two reinforced trials in an NRRN, RNNR pattern. A shock, the minimal current required to interrupt drinking $(0.5 \mathrm{sec}$. at $0.08-$ $0.20 \mathrm{ma}$ ), was delivered through a floor grid $2 \mathrm{sec}$, after the first lick at the spout.

The delayed shock study was conducted simultaneously with the lithium chloride on the same schedule. Non-toxic salty water was the gustatory stimulus. Shock reinforcement was delayed during first trials and gradually increased in intensity $(.05$ to $.30 \mathrm{ma})$ in a schedule designed to produce a drinking pattern during the 20-min. period which resembled that of the corresponding animal drinking toxic salty water.

\section{Results and Discussion}

The results indicate that all reinforcers were effective in producing discrimination learning during the acquisition phase (see Fig. 1), but obvious differences occurred in the post-tests. The avoidance reactions produced by 

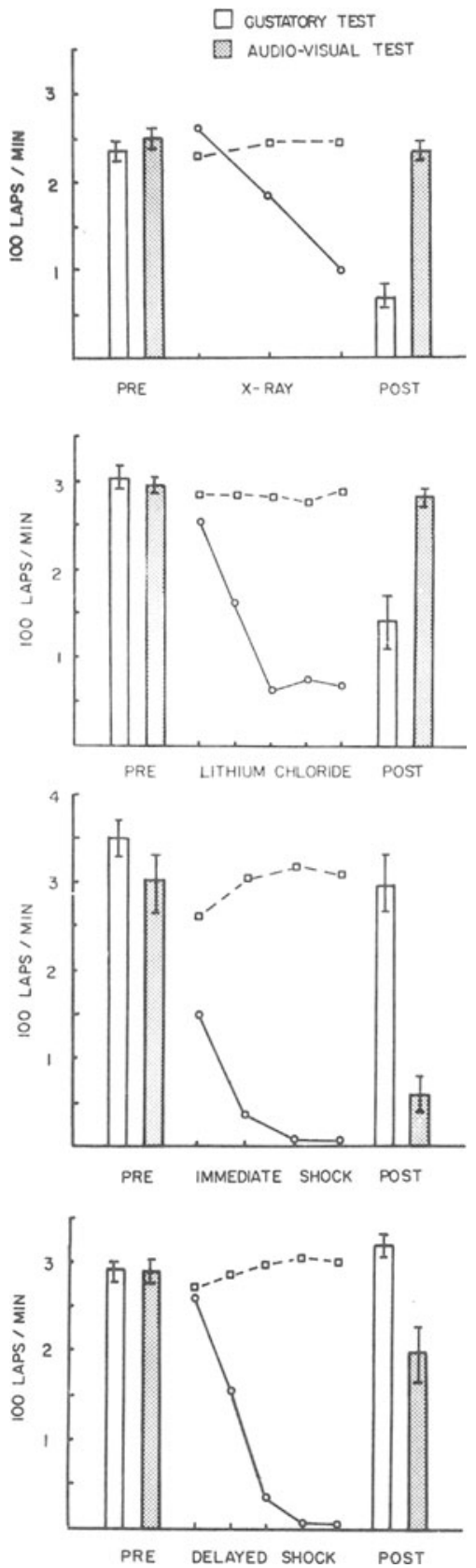

Fig. 1. The bars indicate water intake ( \pm St. Error) during a gustatory test (a distinctive taste) and an audiovisual test (light and sound contingent upon licking) before and after conditional pairing with the reinforcers indicated. The curves illustrate mean intake during acquisition.

$\mathrm{x}$-rays and lithium chloride are readily transferred to the gustatory stimulus but not to the audiovisual stimulus. The effect is more pronounced in the $x$-ray study, perhaps due to differences in dose. The $x$-ray animals received a constant dose while the lithium chloride rats drank a decreasing amount of the toxic solution during training. Nevertheless, the difference between post-test scores is statistically significant in both experiments ( $p<0.01$ by ranks test).

Apparently when gustatory stimuli are paired with agents which produce nausea and gastric upset, they acquire secondary reinforcing properties which might be described as "conditioned nausea." Auditory and visual stimulation do not readily acquire similar properties even when they are contingent upon the licking response.

In contrast, the effect of both immediate and delayed shock to the paws is in the opposite direction. The avoidance reactions produced by electric shock to the paws transferred to the audiovisual stimulus but not to the gustatory stimulus. As one might expect the effect of delayed shocks was not as effective as shocks where the reinforcer immediately and consistently followed licking. Again, the difference between post-test intake scores is statistically significant in both studies $(p<0.01$ by ranks test). Thus, when shock which produces peripheral pain is the reinforcer, "conditioned fear" properties are more readily acquired by auditory and visual stimuli than by gustatory stimuli.

It seems that given reinforcers are not equally effective for all classes of discriminable stimuli. The cues, which the animal selects from the welter of stimuli in the learning situation, appear to be related to the consequences of the subsequent reinforcer. Two speculations are offered: (1) Common elements in the timeintensity patterns of stimulation may facilitate a cross modal generalization from reinforcer to cue in one case and not in another. (2) More likely, natural selection may have favored mechanisms which associate gustatory and olfactory cues with internal discomfort since the chemical receptors sample the materials soon to be incorporated into the internal environment. Krechevsky (1933) postulated such a genetically coded hypothesis to account for the predispositions of rats to respond systematically to specific cues in an insoluble maze. The hypothesis of the sick rat, as for many of us under similar circumstances, would be, "It must have been something I ate."

\section{References}

Bamett, S. A. The rat: a study in behavior.Chicago: Aldine Press, 1963.

Garcia, J., \& Koelling, R. A. A comparison of aversions induced by $x$-rays, toxins, and drugs in the rat. Radiat. Res., in press, 1965.

Haley, T. J., \& Snyder, R. S. (Eds.) The response of the nervous system to ionizing radiation. Boston: Little, Brown \& Co., 1964

Krechevsky, I. The hereditary nature of 'hypothesis'. J. comp. Psychol., 1932, 16, 99-116.

Nachman, M. Learned aversion to the taste of lithium chloride and generalization to other salts. J. comp. physiol. Psychol., 1963, $56,343-349$

\section{Note}

1. This research stems from doctoral research carried out at Long Beach V. A. Hospital and supported by NIH No. RH00068. Thanks are extended to Professors B. F. Ritchie, D. Krech and E. R. Dempster, U. C. Berkeley, California. 